\title{
CLUSTER MODEL OF CHARGE TRANSFER EXCITONS IN MOLECULAR CRYSTALS. II. LOCAL FRENKEL EXCITONS IN DOPED CRYSTALS
}

\begin{abstract}
A. EILMES
K. Gumiński Department of Theoretical Chemistry, Jagiellonian University

Ingardena 3, 30-060 Kraków, Poland

(Received March 20, 1993; in final form May 25, 1993)

Charge transfer induced local/resonance Frenkel exciton states in doped naphthalene crystals are investigated in terms of the cluster model, proposed in Paper I of this series. The effect of cluster size is studied in order to assess the physical relevance of the results. Possible methods of accounting for the random arrangement of the impurities are suggested and discussed.
\end{abstract}

PACS numbers: $71.35 .+z$

\section{Introduction}

It has been recently shown [1-4] that when the naphthalene crystal is doped with some benzene derivatives, local Fronkcl exciton states are produced. The dependence of the energy of the lowest local state on the ionization potential of the dopant suggests that their formation is due to off-diagonal interactions between the Frenkel and charge transfer excitons. This dependence was interpreted in terms of a dimer model [3] and subsequently in terms of a (more advanced) cluster model [4], proposed originally in Paper I of this series [5].

Although semiquantitative agreement with experiment was obtained, the results were found to depend on the size of the cluster used in the calculations. This feature of the cluster model becomes more important when one is interested in the structure of multiple local levels induced by the same impurity molecule.

The present paper is devoted to the study of the effect the cluster size has on the predicted pattern of local states, with the aim to suggest a reasonable method of simulating the situation in an actual crystal, and in particular to account for the fact that the crystal contains many dopant molecules. 


\section{Calculations}

The doped naphthalene crystal is represented by a two-dimensional cluster where the molecules are arranged in $m$ rows and $n$ columns, and one of the molecules is the impurity. The basis set consists of the wave functions representing the localized Frenkel excitons and localized charge transfer states. These localized states are coupled by resonance, charge transfer and exciton dissociation integrals. For all kinds of interactions the nearest-neighbour approximation is adopted. For the details the Reader is referred to Refs. [4-6].

The parametrization is the same as described in Ref. [6]. The calculations refer to the energy range of the $A^{\prime}$ transition of the neat naphthalene crystal, which is a vibronic replica of the lower energy Davydov component of the lowest Frenkel state.

The calculations were done for the whole series of impurities listed in [6] (where also the complete set of experimental results can be found). As the objective of this paper is to compare the results for various cluster sizes and not to interpret the pattern of local levels for individual impurities, only some representative cases will be presented.

\section{Small cluster's}

When applying the clustcr model to the simulation of the doped crystal structure the question arises how large clusters should be used. On the one hand it is convenient to use small clusters, containing about 100 molecules, which reduces memory requirements and computation time. Another advantage of this choice is that the dopant concentration in the cluster is close to that in the pre-sublimation mixture $(1 \%,[6])$, and presumably to the actual concentration in the crystals for which experimental data are available. Ilowever, small density of states in small clusters may produce instability of the results.

The calculations were performed for individual clusters containing from 64 to 144 molecules $(8 \times 8,9 \times 8,8 \times 10,10 \times 8,11 \times 8,9 \times 10,8 \times 12,12 \times 8,10 \times 10$, $9 \times 12,11 \times 10,12 \times 10,10 \times 12,11 \times 12,12 \times 12)$. Figures $1 \mathrm{a}-\mathrm{d}$ present the results for the impurities whose vertical ionization potential is significantly lower $\left(1,4\right.$-toluidine, $I_{\mathrm{imp}}=7.81 \mathrm{eV}$ ), slightly lower (aniline, $I_{\mathrm{imp}}=8.04 \mathrm{eV}$ ), slightly higher (hydroquinone, $I_{\mathrm{imp}}=8.44 \mathrm{eV}$ ) and significantly higher (toluene, $I_{\mathrm{imp}}=$ $8.78 \mathrm{eV})$ than that of naphthalene $\left(I_{\text {naph }}=8.22 \mathrm{eV}\right)$. The results are presented for selected representative cluster sizes $(8 \times 8,8 \times 10,10 \times 10,12 \times 10,12 \times 12$; the $10 \times 10$ size corresponds to the dopant concentration in the pre-sublimation mixture [6]).

Each state obtained from the calculations is represented by a vertical bar in the lower part of the panel. The length of the corresponding bar in the upper part of the panel is proportional to the intensity (in arbitrary units) of the optical transition from the ground state to that state.

The group of very intense states at about $3.957 \mathrm{eV}$ corresponds to the $A^{\prime}$ band of the neat crystal. The intensities of those states are not drawn to scale. The broken lines show the positions of the experimentally observed local states 

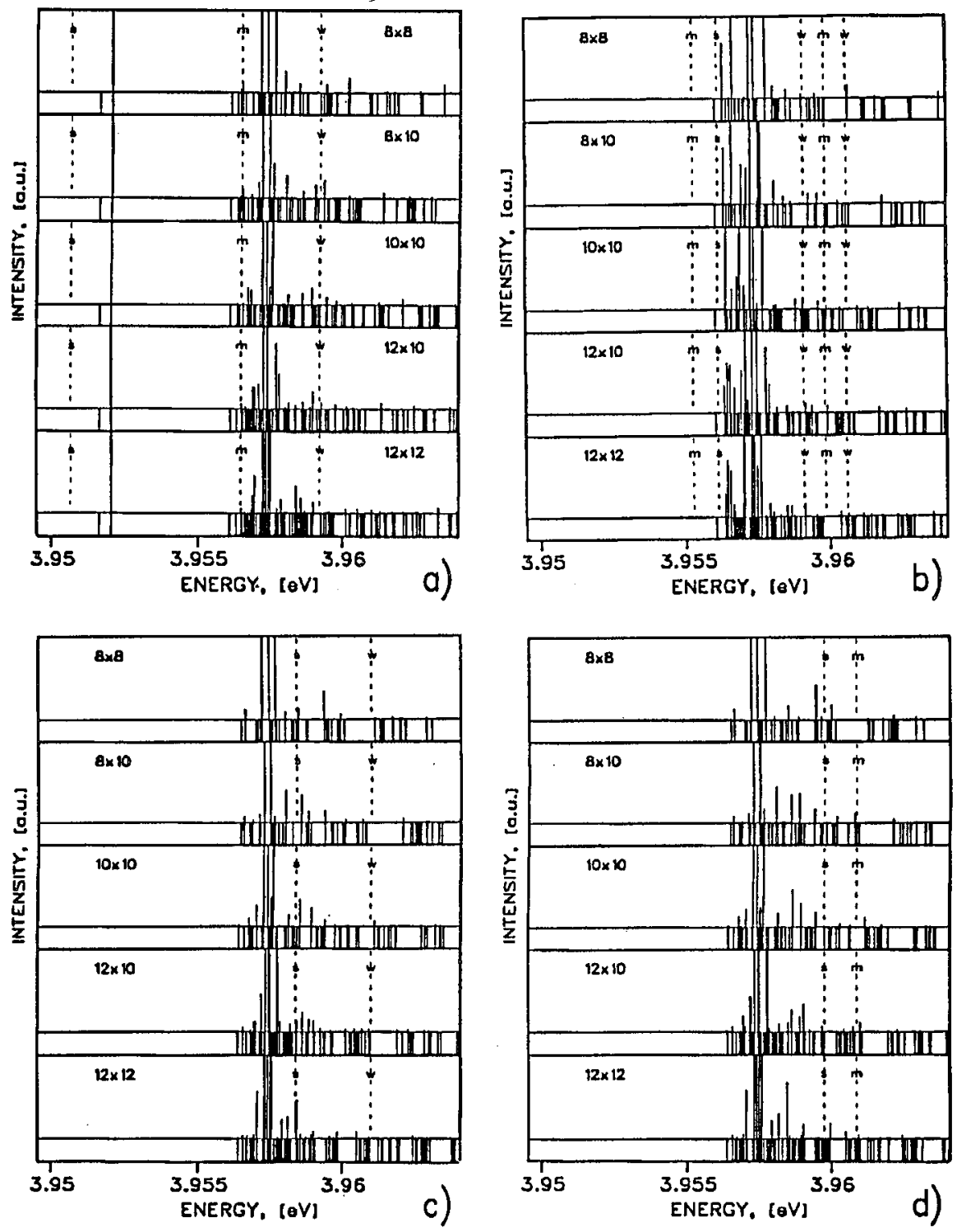

Fig. 1. The calculated pattern of energy levels and absorption intensities for small clusters containing one molecule of a) 1,4-toluidine; b) aniline; c) hydroquinone; d) toluene. The broken lines represent the experimental results from Ref. [6], the letter code indicating the intensities. The lower part of each panel shows the density of states; the upper part - absorption intensities (sce text). 
(with intensities labelled in the standard way). $\Lambda$ s cluster size effects are the sole object of this paper, we will not discuss the discrepancies between the calculated and the observed spectra. That analysis can be found in Ref. [6].

Even a cursory glance at the plots makes it clear that the energy of the local states deep below the $A^{\prime}$ band is insensitive to the cluster size. That state is an example of a "genuine" local state (charge transfer induced local Frenkel exciton state - CTILFES).

This indicates that as far as the CTILFES are concerned, calculations for relatively small clusters are sufficient, provided the cluster size and shape allow the impurities to be separated by at least a couple of host molecules (in order to avoid multi-dopant interactions). In fact, even the smallest of the sizes considered above is large enough.

The picture is more complicated for the states near and above the $A^{\prime}$ transition, which are the "resonance" states (charge transfer induced resonance Frenkel exciton states - CTIRFES). The stability of the results is obviously poor - there are significant changes in the energy and intensity of the resonance states.

This behaviour may have two reasons. The first one is insufficient density of states. While in an infinite crystal the energy levels form a continuum (for all possible wave vector values), in the cluster calculations for the neat crystal only a relatively small number (depending on the cluster size and corresponding to a discrete set of permitted wave vector values) of energy levels is allowed by the periodic boundary conditions. The breakdown of the translational symmetry of the cluster due to the impurity molecule activates some of those levels in the optical spectrum, but barely affects their energies. In effect, the energy pattern of the resonance levels is governed by the symmetry and size of the cluster, which produces differences for various cluster sizcs.

The second possibility is that the poor stability of the results is not merely a kind of numerical artefact but represents a genuine physical effect. Due to the periodic boundary conditions, some of the interactions between different dopant molecules via the host crystal are implicitly included. They are very weak (since the impurities are far away from each other), hence they do not influence the CTILFES, but may still affect the resonances which are much more sensitive even to small variations of the interactions. Therefore, the energy shifts and intensity changes of the CTIRFES may correspond (at least partly) to actual changes of the inter-impurity interaction, and in this way indirectly reflect the local variations of the dopant concentration.

\section{Large clusters}

It seems that the most natural way to improve the stability of the results for resonance states consists in performing the calculations for larger clusters, since in that case the density of cluster stat is increased and the inter-impurity interactions are suppressed due to larger distances between dopant molecules. (It should be noted that this approach has its disadvantages as wcll: a considerable increase of the computation time and loss of the direct relation of the size of the model cluster to the actual impurity concentration in the crystal). 
Therefore, the calculations were repeated for a series of clusters containing from 256 to 342 molecules $(16 \times 16,17 \times 16,18 \times 16,16 \times 18,19 \times 16,17 \times 18,20 \times 16$, $16 \times 20,18 \times 18,17 \times 20$ and $19 \times 18$ ), in eacl case one of the molecules being the impurity. The results for some representative sizes are shown in Figs. 2a,b for two different dopants - with low (aniline $-I_{\text {imp }}=8.04 \mathrm{eV}$ ), and with high (toluene $-I_{\mathrm{imp}}=8.78 \mathrm{eV}$ ) ionization potential.
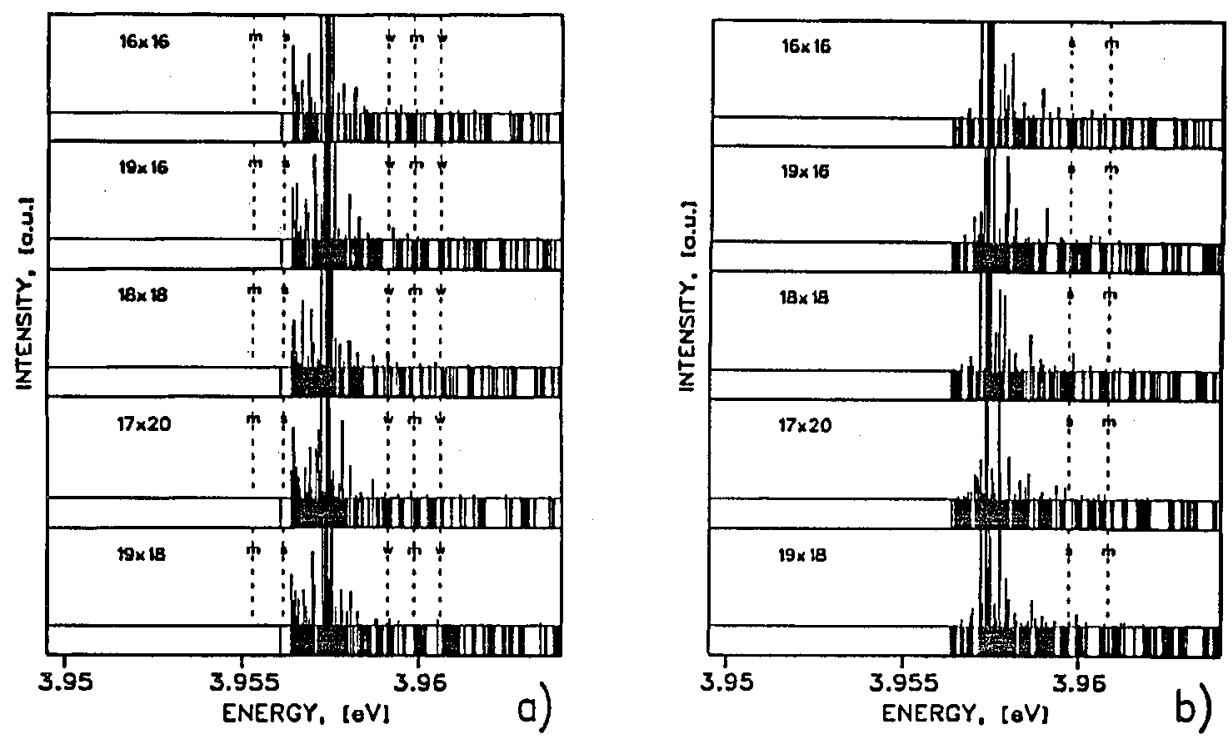

Fig. 2. The calculated pattern of energy levels and absorption intensities for large clusters containing one molecule of a) aniline; b) toluene. The lines have the same meaning as in Fig. 1.

It is readily seen that although the differences between clusters of different size still remain, the general picture is much more stable. The local levels of non-negligible intensity tend to group in some well-defined energy ranges, which allows one to discern a number of characteristic "bands". At the same time, the relative intensity of the local levels decreases, as the average dopant concentration for large clusters is only about $0.3 \%$.

This causes the objection that the results can hardly be physically relevant for a crystal with a different (1\%) dopant concentration which is of experimental interest. The objection may be a crucial one if the pattern of the resonances is determined primarily by the interaction between impurities, which may well be the case.

\section{Averaging}

For the reason presented above, the calculations for large clusters are not very likely to be physically relevant. This motivates a search for a method of improving the stability of the results for small clusters. 
It may be based on the following argument:

Although for one particular cluster shape and size only some particular points ( $k$ values) from the Brillouin zone of the infinite crystal are probed, they are generally different for another shape and/or size. It suggests that an average of the results over a series of clusters would allow one to explore larger domains of the Brillouin zone and hence it should provide a more adequate description than the results for any individual cluster. At the same time, the average dopant concentration may be maintained at any desired level by a judicious choice of cluster sizes. The additional advantage of this approach consists in the fact that it automatically takes into account some degrees of randomness of inter-impurity distances in a real crystal.

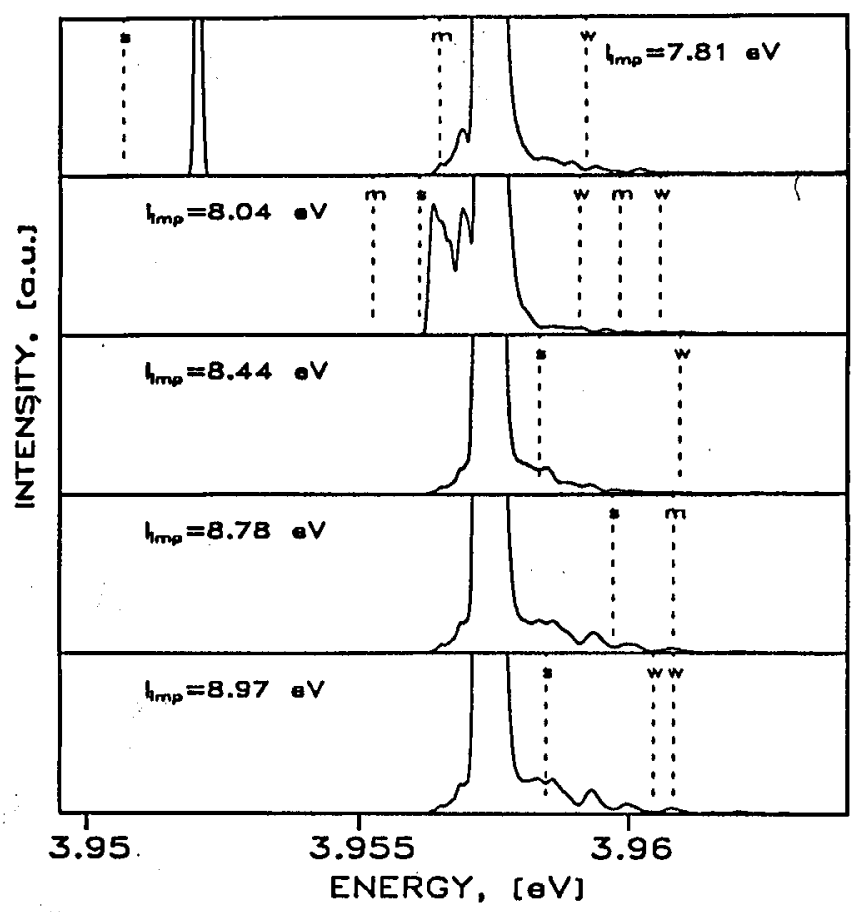

Fig. 3. The calculated absorption spectrum averaged over a series of small clusters for crystals doped with (from top to bottom) 1,1-toluidine, aniline, hydroquinone, toluene, and 1,2-dibromobenzene.

In this work, the above general idea was implemented by averaging over the whole series of small clusters described in Sec. 3. In effect, the mean dopant concentration was $1 \%$, and reflected the impurity concentration in the pre-sublimation mixture.

Each predicted level was represented by a Gaussian curve. The width of the Gaussian was $\sigma=5 \times 10^{-5} \mathrm{eV}$ below the $A^{\prime}$ band of the neat crystal and was increased to $\sigma=1 \times 10^{-4} \mathrm{eV}$ above it, in order to imitate the broadening of the 
bands due to new lattice phonon continua opening with the new vibronic transition in the intramolecular mode. *

Figure 3 shows the results, for five impurities covering the investigated range of ionization potentials. The general picture becomes much clearer than for any of the individual clusters.

For the dopant ionization potential lower than $I_{\text {naph }}(8.22 \mathrm{eV})$ two bands below the $A^{\prime}$ transition are easily distinguishable.

The resonance states above the $A^{\prime}$ band are less well pronounced. Nevertheless, for the impurity ionization potential higher than that of naphthalene, a series of bands of substantial intensity appears. The basic set of resonance levels is the same for all those dopants. The positions of the individual states remain almost unchanged upon an increase of $I_{\mathrm{imp}}$, while their intensity shifts to higher energies.

\section{Conclusions}

Generally, the presented material suggests that for the interpretation of the spectrum of a real crystal containing many dopant molecules, calculations for only one particular cluster could not provide satisfactory results. Small clusters correctly account for the dopant concentration and allow one to speed up the calculations, but due to insufficient density of states the results are not stable. Large clusters offer better stability (will larger numerical effort) but fail to account for some features of the inter-impurity interactions in the crystal. This is due to incorrect distances between dopant molecules, resulting from too low impurity concentration. Moreover, in an actual crystal the impurities are distributed more or less randomly, which is in no way reflected in the single-cluster model.

One of the possible methods of dealing with the random distribution of impurities is the averaging of the results over a set of individual clusters. Then, if the size of the clusters is judiciously cliosen (i.e. relatively small), the mean dopant concentration is correctly reproduced, and at the same time the distribution of cluster sizes mimics, at least to some extent, the statistical fuctuations of the distance between dopant molecules. The additional advantage is that the averaged spectra are clearer and easier to interpret.

The drawbacks of this approach are twofold.

Firstly, the calculations for neat clusters $[5,8]$ indicate that the density of states, even if averaged over a large set of small clusters, still exhibits some gaps and discontinuities, unlikely to occur in an infinite crystal. It seems that this problem is inherent to the finite cluster approach and cannot be avoided - it can only be reduced by using larger clusters.

Secondly, there is no systematic but straightforward way of improving the results by extending the set of clusters included in the averaging. In fact, if the set is extended by including much larger clusters, the concentration constraint would

*The smallest reasonable values of the Gaussian width are preferred in order to reduce the diffuseness of weak states. The specific values used in the calculations were chosen in such a way as to reproduce roughly the typically observed band widths of the $A^{\prime}$ transition and of the well-separated CTILFES peaks [7]. It is worth noting that the effect of changing the Gaussian width is similar to that of changing the impurity concentration. 
require that also much smaller clusters should be included, and for those the model is no longer physically relevant due to much more pronounced boundary effects.

In this respect, another difficulty is bound to emerge for ligher dopant concentrations (which are also of experimental interest $[9,10]$ ), since the cases, where two or more impurity molecules are close to each other, would be practically impossible to handle in that way.

In view of this, the natural alternative is to perform the calculations for a cluster of the maximum technically feasible size, containing not one but many randomly distributed dopant molecules. The concentration of the impurity could be controlled by changing the probabilities of liost and dopant site occupancy. Then, the results should be averaged over a scries of clusters which would all have the same size but would differ in the (random) arrangement of impurity molecules. The possibility of controlling the average dopant concentration in the sample set of clusters will allow one to study the concentration effects, in particular the structure of CTILFES in heavily doped crystals, where the configurations of some impurity molecules spaced by only one or two lattice spacings, give rise to new peaks.

This kind of simulations is beyond the scope of this work and is taken up in other papers to which the Reader is referred for details $[6,11]$.

\section{Acknowledgments}

This research was supported by the grant No. $206699101(1723 / 2 / 91)$ from the Committee for Scientific Research (Poland). The author expresses his gratitude to Prof. Piotr Petelenz for his valuable comments and helpful discussions.

\section{R.eferences}

[1] I.V. Brovehenko, N.I. Ostapenko, V.I. Sugakov, M.T. Shpak, Izv. Acad. Nauk. SSSR Ser. Fiz. 52, 659 (1988).

[2] I.V. Brovchenko, N.I. Ostapenko, M.T. Slıpak, Fiz. Tverd. Tela 30, 261 (1988).

[3] P. Petelenz, I.V. Brovchenko, D. Mucha, M. Slawik, Chem. Phys. 143, 115 (1990).

[4] P. Petelenz, A. Eilmes, Chern. Phys. 155, 247 (1991).

[5] P. Petclenz, A. Eilmes, Acta Phys. Pol. A 70, 727 (1991).

[6] I.V. Brovchenko, A. Eilmes, P. Petelenz, J. Chem. Phys. 98, 3737 (1993).

[7] I.V. Brovchenko, unpublished results.

[8] A. Eilmes, unpublished results.

[9] N.I. Ostapenko, V.I. Sugakov, M.T. Shpak, J. Lumin. 4, 261 (1971).

[10] N.I. Ostapenko, V.I. Sugakov, M.T. Shpak, Spectroscopy of Defects in Molecular Crystals, Naukova Dumka, Kiev 1988 (in Russian).

[11] A. Eilmes, P. Petelenz, in preparation. 